\title{
Long intergenic noncoding RNA 00707 promotes colorectal cancer cell proliferation and metastasis by sponging miR-206
}

This article was published in the following Dove Press journal: OncoTargets and Therapy

\author{
Huifang Zhu ${ }^{\mathrm{I}-3}$ \\ Guoyang $\mathrm{He}^{\mathrm{l}-3}$ \\ Yongqiang Wang ${ }^{1-3}$ \\ Yuhan $\mathrm{Hu}^{1-3}$ \\ Zheying Zhang ${ }^{1-3}$ \\ Xinlai Qian ${ }^{1-3}$ \\ Yongxia Wang ${ }^{1-3}$
}

'Department of Pathology, Xinxiang Medical University, Xinxiang City 453000,

Henan, People's Republic of China; ${ }^{2}$ Department of Pathology, The Third Affiliated Hospital of Xinxiang Medical University, Xinxiang City 453000, Henan, People's Republic of China; ${ }^{3}$ Opening Laboratory for Key Discipline of Tumor Reversal Molecular Biology of Henan Higher Education, Xinxiang Medical University, Xinxiang City 453000, Henan, People's Republic of China
Correspondence: Xinlai Qian; Yongxia Wang

Department of Pathology, Xinxiang Medical University, No.60I Jinsui Street, Xinxiang 453000, Henan, People's Republic of China

$\mathrm{Tel} / \mathrm{Fax}+8603733029620$

Email qxIfssws@163.com;

xxmusfyblkwyx@sohu.com
Background: The incidence and mortality of colorectal cancer (CRC) are rising worldwide. Long-noncoding RNAs (lncRNAs) are known to play key roles in the development of human cancers, including CRC. However, the function and underlying mechanism of long intergenic noncoding RNA 00707 (LINC00707) in the development of CRC are unknown.

Materials and methods: The expression of LINC00707 and miR-206 in tissue samples or cell lines was measured by quantitative reverse transcription PCR (qRT-PCR). The protein expression of neurogenic locus notch homolog protein 3 (NOTCH3) and transmembrane 4 L6 family member 1 (TM4SF1) was assessed by Western blotting. Cell proliferation, migration, and invasion were assessed by the 3-(4,5-dimethylthiazol-2-yl)-2,5-diphenyltetrazolium bromide (MTT) and transwell assays. Luciferase reporter assay and biotin-coupled miRNA capture assay were used to explore the relationship between LINC00707 and miR206 expression.

Results: The expression of LINC00707 was significantly upregulated in CRC tissues as compared with the adjacent non-CRC tissues. LINC00707 expression was significantly correlated with tumor size, lymphatic metastasis, and distant metastasis, but not significantly correlated with age and gender. Knockdown of LINC00707 expression significantly inhibited LoVo and HCT116 cell proliferation, migration, and invasion. LINC00707 acted as a molecular sponge by competing for miR-206 and indirectly modulating the expression of its targets, NOTCH3 and TM4SF1.

Conclusion: LINC00707 promotes CRC cell proliferation and metastasis by sponging miR206, suggestive of its potential application for CRC treatment.

Keywords: long intergenic noncoding RNA 00707, colorectal cancer, miR-206, NOTCH3, TM4SF1

\section{Introduction}

Colorectal cancer (CRC) is the third most commonly diagnosed malignancy and the second leading cause of cancer-related mortality worldwide. ${ }^{1}$ CRC incidence and associated mortality rates are gradually rising in China. ${ }^{2}$ Despite tremendous efforts for improvement in the clinical treatment of $\mathrm{CRC}$, the 5-year overall survival rate of CRC is unsatisfied owing to cancer recurrence and metastasis. ${ }^{3}$ Therefore, elucidation of the molecular mechanism underlying the progression of $\mathrm{CRC}$ is desirable for the development of new treatment strategies and improvement of patient prognosis.

Long-noncoding RNAs (lncRNAs) are transcripts that are more than 200 nucleotides in length without or with limited protein-coding capacity. ${ }^{4-6}$ LncRNAs play 
essential roles in several biological processes, including embryonic development, cell growth and differentiation, and tumorigenesis. ${ }^{7}$ Therefore, lncRNAs may contribute to the development and progression of various human diseases, including cancers. ${ }^{7-10}$ Long intergenic noncoding RNA 00707 (LINC00707) is a 3,087-bp noncoding RNA located on chromosome 10p14. The knockdown of LINC00707 expression was shown to repress lung adenocarcinoma cell proliferation and migration. ${ }^{11}$ Wang et al found that LINC00707 promotes hepatocellular carcinoma (HCC) progression. ${ }^{12}$ However, the function and underlying mechanism of LINC00707 in the development of CRC remain to be elucidated.

Although the knowledge on the molecular mechanisms of lncRNAs still remains limited, more and more reports reveal that LncRNAs function as competing endogenous RNAs (ceRNAs) or natural microRNA (miRNA) sponges, thereby relieving the repression of target mRNAs. ${ }^{13}$ miRNAs are small non-coding RNAs within a size range of 21-25 nucleotides, repress the translation of their target mRNAs or degrade mRNAs. ${ }^{14}$ However, whether LINC00707 functions as ceRNA of miRNAs to regulate tumor progression in CRC remains to be investigated.

In the present study, the expression of LINC00707 in CRC tissues was measured and the relationship between LINC00707 expression and clinicopathologic characteristics of CRC patients was analyzed. Functional effects of LINC00707 on CRC cell growth and metastasis were assessed in vitro. Moreover, the underlying mechanism of LINC00707 functions in CRC was investigated by confirming whether LINC00707 functions as ceRNA of miR-206 to thereby relieve the translation repression of target mRNA, neurogenic locus notch homolog protein 3 (NOTCH3) and transmembrane 4 L6 family member 1 (TM4SF1). ${ }^{15,16}$

\section{Materials and methods}

\section{Clinical tissue samples}

A total of $40 \mathrm{CRC}$ tissues and paired adjacent tissue samples (at least $5 \mathrm{~cm}$ away from the tumor tissues) were collected by surgical resection from patients with CRC who had received neither chemotherapy nor radiotherapy at the Third Affiliated Hospital of Xinxiang Medical University Hospital. The procedures of this study were approved by the Ethics Committee of Third Affiliated Hospital of Xinxiang Medical University. Written informed consent was received from all participants in accordance with the Declaration of Helsinki. The diagnosis and clinicopathological characteristics included age, gender, tumor size, lymph node metastasis, and distant metastasis, which were confirmed by two pathologists (Table S1). All the tissue samples were immediately frozen in liquid nitrogen and stored at $-80^{\circ} \mathrm{C}$ until RNA extraction.

\section{Quantitative reverse transcription PCR (qRT-PCR)}

Total RNA from tissues or cultured cells was separated using Trizol reagent (Invitrogen, Carlsbad, CA, USA) according to the manufacturer's instructions. RNA $(1 \mu \mathrm{g})$ was reverse transcribed to cDNA with reverse transcription kit (Takara, Dalian, China). qRT-PCR was performed on ABI 7500 RT-PCR system (Applied Biosystems, Foster City, CA, USA) with SYBR $^{\circledR}$ Premix Ex Taq ${ }^{\mathrm{TM}}$ Kit (TaKaRa) and Mir-X ${ }^{\mathrm{TM}}$ miRNA qRT-PCR SYBR $^{\circledR}$ Kit (Clontech Laboratories, Inc., Mountain View, CA, USA) in accordance with the manufacturer's protocol. The primers were synthesized by GenePharma (Shanghai, China) and the primer sequences were as follows: LINC00707 forward 5'-CCAACAGGGTATCAGAATTCTC-3' and reverse 5'-TGCTGACAATAGCCATTAGG-3'; $\beta$-actin forward 5'-TGGATCAGCAAGCA GGAGTA- ${ }^{\prime}$ ' and reverse 5'-TCGGCCACATTGTGAACTTT-3'; miR-206 forward, 5'-CAGATCCGATTGGAATGTAAGG-3' and reverse, 5'TATGCTTGTTCTCGTCTCTGTGTC-3'; U6 forward 5'CTCGCTTCGGCAGCACA-3' and reverse 5'-AACGCT TCACGAATTTGCGT-3'. The expression of LINC00707 and miR-206 was normalized to the expression of $\beta$-actin and U6, respectively. Data were analyzed by comparative cycle threshold $(\mathrm{CT})\left(2^{-\Delta \Delta \mathrm{CT}}\right)$ method. All reactions were performed in triplicates.

\section{Cell culture and transfection}

The human CRC cell lines LoVo and HCT116 were purchased from the Shanghai Institutes of Biological Sciences (Shanghai, China). Cells were maintained in Dulbecco's modified Eagle medium (DMEM; HyClone, Logan, UT, USA) supplemented with $10 \%$ fetal bovine serum (FBS; HyClone) and 1\% penicillin-streptomycin (Thermo Scientific, Waltham, MA, USA) and cultivated at $37^{\circ} \mathrm{C}$ in $5 \% \mathrm{CO}_{2}$. LINC00707 siRNA (si-LINC00707 $1^{\#}:$ 5'-GGCUUUCCAUGACCCAUAAUU-3', si-LINC $007072^{\#}:$ 5'-GGAAGCCACU CCUGCAUUUUU-3', si-LINC00707 $3^{\#}: \quad 5^{\prime}$-GCAGGAACAUCACCAUCUU UU-3'), siRNA negative control (si-NC), miR-206 mimic, and negative control (NC) were all purchased from GenePharma (Shanghai, China). Lipofectamine ${ }^{\mathrm{TM}}$ 
2000 (Invitrogen) was used for cell transfection according to the manufacturer's instructions.

\section{Cell proliferation assay}

Cell proliferation was determined with the 3-(4,5-dimethylthiazol-2-yl)-2,5-diphenyltetrazolium bromide (MTT) assay. In brief, $2 \times 10^{3}$ cells were seeded in each well of a 96 -well plate in $100-\mu \mathrm{L}$ volume in triplicates. At 24, 48, and $72 \mathrm{hrs}$ after transfection, MTT (10 $\mu \mathrm{L}, 5 \mathrm{mg} / \mathrm{mL}$ ) was added into each well and incubated for $4 \mathrm{hrs}$ at $37^{\circ} \mathrm{C}$. After incubation, $100 \mu \mathrm{L}$ of dimethyl sulfoxide (DMSO) was added to solubilize the formazan. The absorbance was measured at $570 \mathrm{~nm}$ wavelength with a microplate reader (Bio-Rad, Hercules, CA, USA).

\section{Transwell migration and invasion assay}

Cell migration and invasion were evaluated using the BD 24-well Transwell chamber (BD Biosciences, San Jose, CA, USA). Transfected cells $\left(1 \times 10^{5}\right)$ were seeded in serum-free medium in the top chamber of each well. Matrigel (BD Biosciences) was used to cover the top side of the membrane for invasion assay and Matrigelfree condition was used for migration assay. The lower chamber was filled with complete medium supplemented with $10 \%$ FBS. The cells were incubated at $37^{\circ} \mathrm{C}$ with $5 \%$ $\mathrm{CO}_{2}$ for $48 \mathrm{hrs}$, and the non-traversed cells were removed from the upper filter with a cotton swab. Next, the ratio of cells for migration and invasion assays was determined using two methods, crystal violet staining and MTT assay. For crystal violet staining, the cells that migrated or invaded to the reverse side of chamber inserts were fixed with methanol for 30 mins and stained with crystal violet for 15 mins. The number of migrated or invasive cells were determined by counting the stained cells in ten randomly selected fields under a microscope (Olympus, Tokyo, Japan). For MTT assay, $500 \mu \mathrm{L}$ complete medium containing $0.5 \mathrm{mg} / \mathrm{mL}$ MTT was added into the lower chamber of each well. The top chamber was immersed in the medium and incubated for $4 \mathrm{hrs}$ at $37^{\circ} \mathrm{C}$. After incubation, $100 \mu \mathrm{L}$ of DMSO was added to solubilize the formazan. The absorbance was measured at $570 \mathrm{~nm}$ wavelength with a microplate reader (Bio-Rad, Hercules, CA, USA). All experiments were performed in triplicates.

\section{Western blot analysis}

Cellular proteins were extracted with a lysis buffer supplemented with a protease inhibitor phenylmethanesulfonyl fluoride (PMSF; Beyotime, Shanghai, China). Protein concentration was determined with the bicinchoninic acid (BCA) protein assay kit (Beyotime). Equivalent amounts of denatured proteins $(20 \mu \mathrm{g})$ were separated with sodium dodecyl sulfate-polyacrylamide gel electrophoresis and transferred onto polyvinylidene fluoride membranes (Millipore, Billerica, MA, USA). After blocking, the membranes were incubated at $4^{\circ} \mathrm{C}$ overnight with following primary antibodies: anti-NOTCH3 (dilution 1:1,000, Cell Signaling Technology), anti-TM4SF1 (dilution 1:1,000, Thermo Scientific), and anti-glyceraldehyde-3-phosphate dehydrogenase (GAPDH) (dilution 1:5,000; Abcam). After washing with Tris-buffered saline containing Tween-20 (TBST), the membrane was incubated with a horseradish peroxidase (HRP)-labeled secondary antibody (dilution 1:5,000, Boster, Wuhan, China) for $2 \mathrm{hrs}$ at $25^{\circ} \mathrm{C}$, followed by washing with TBST. The immunolabeled proteins were reacted with chemiluminescent HRP substrate (Thermo Scientific, Waltham, MA, USA).

\section{Bioinformatic analysis}

The potential binding sites of miRNAs on LINC00707 were predicated using lncRNABase (http://starbase.sysu. edu.cn/agoClipRNA.php?source=lncRNA).

\section{Luciferase reporter assay}

LINC00707 wild-type (WT) with potential miR-206binding sites or mutant (MUT) of each sites were generated and fused to a luciferase reporter vector psi-CHECK-2 (Promega, Madison, WI, USA) to analyze the interaction between LINC00707 and miR-206. The binding site of miR206 on LINC00707 was mutated into AGATAGGA. HEK293T cells were co-transfected with luciferase plasmids and miR-206 mimic or miRNA negative control. After 48 hrs transfection, luciferase activities were detected with a Dual-Luciferase Reporter Assay System (Promega) according to the manufacturer's instructions. The ratio of firefly to Renilla luciferase activity was assessed.

\section{Biotin-coupled miRNA capture assay}

The biotin-coupled miRNA capture assay was performed as previously described. ${ }^{17}$ In brief, miR-206 mimics and miRNA negative control were labeled with biotin at the $3^{\prime}$ end and transiently transfected into LoVo and HCT116 cells. After 24 hrs of culture, the cells were harvested and lysed in a lysis buffer, followed by incubation with streptavidin beads (Thermo Scientific) to pull-down the biotin-coupled RNA complex. After washing with lysis buffer, TRIzol reagent (Invitrogen) was used to recover the RNAs that 
specifically interacted with miRNA. The abundance of LINC00707 in the bound fraction was measured with qRTPCR after its reverse transcription to cDNA.

\section{Statistical analysis}

All statistical analyses were performed using the Statistical Package for the Social Sciences version 19.0 software (SPSS Inc., Chicago, IL, USA). All data are expressed as the mean \pm standard deviation (SD). Student's $t$-test and one-way analysis of variance were carried out to evaluate significant differences. Statistical significance was considered at $P<0.05$.

\section{Results}

\section{LINC00707 expression is upregulated in CRC tissues}

The expression of LINC00707 in 40 CRC tissues and paired adjacent non-CRC tissues was detected with qRTPCR analysis (Figure 1). The expression of LINC00707 was significantly upregulated in CRC tissues as compared with the adjacent non-CRC tissues.

\section{LINC00707 expression correlates with clinicopathologic characteristics of patients with CRC}

The relationship between various clinicopathological characteristics of patients with CRC and LINC00707 expression was analyzed (Figure 2). Age and gender did not show significant correlation with LINC00707 expression, while tumor size, lymphatic metastasis, and distant metastasis showed significant correlation with LINC00707 expression.

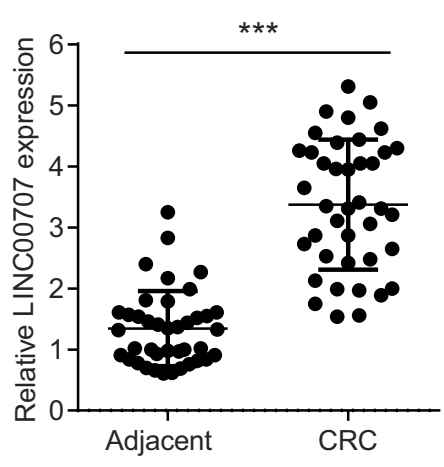

Figure I Expression of LINC00707 in colorectal cancer (CRC) tissues and paired adjacent non-colorectal cancer tissues was detected by quantitative reverse transcription PCR analysis $(* * * P<0.001)$.

\section{Knockdown of LINC00707 inhibits cell proliferation, migration, and invasion in LoVo and HCTII 6 cells}

To investigate whether LINC00707 expression has functional impacts on CRC cells, the expression of LINC00707 in two CRC cell lines (LoVo and HCT116) was silenced by transfection with LINC00707 siRNA (si-LINC00707). qRTPCR results showed that the expression of LINC00707 in both LoVo and HCT116 cells was downregulated upon transfection with si-LINC00707, and the silencing effect of si-LINC00707 $3^{\#}$ was significantly higher than that of siLINC00707 $1^{\#}$ and si-LINC00707 2 $2^{\#}$ (Figure 3A). Thus, siLINC00707 $3^{\#}$ was selected for further functional assays.

The effects of LINC00707 knockdown on LoVo and HCT116 cells were investigated. MTT and transwell assay results demonstrate that LINC00707 knockdown significantly inhibited the proliferation (Figure $3 \mathrm{~B}$ and $\mathrm{C}$ ), migration (Figure $3 \mathrm{D}$ and $\mathrm{E}$ ), and invasion (Figure $3 \mathrm{~F}$ and G) of both LoVo and HCT116 cells.

\section{LINC00707 acts as a molecular sponge for miR-206 and indirectly modulates the expression of its target proteins, NOTCH3 and TM4SFI}

LncRNAs have the ability to directly bind to miRNAs and function as a molecular sponge. We investigated whether LINC00707 exerts similar functions. Bioinformatics analysis revealed that the binding sites of miR-206 on LINC00707 sequence are more than other miRNAs, which are downregulated in CRC tissues and suppress tumor progression in CRC cells. So miR-206 was selected for the further study. The putative binding sites between LINC00707 and miR-206 were shown in Figure 4A. The results of the dual-luciferase reporter assay showed that the cells co-transfected with WT LINC00707 and miR-206 mimic exhibited low luciferase activity, while those cotransfected with MUT LINC00707 and miR-206 mimic had no obvious inhibitory effect on luciferase activity (Figure 4B). In addition, biotin-coupled miRNA capture assay results indicated that LINC00707 level was higher in biotin-miR-206 transfected LoVo and HCT116 cells as compared with the cells treated with biotin-miR-NC (Figure 4C). qRT-PCR results showed that the expression level of miR-206 increased in both LoVo and HCT116 cells transfected with si-LINC00707 as compared with those transfected with si-NC (Figure 4D). Western blot 

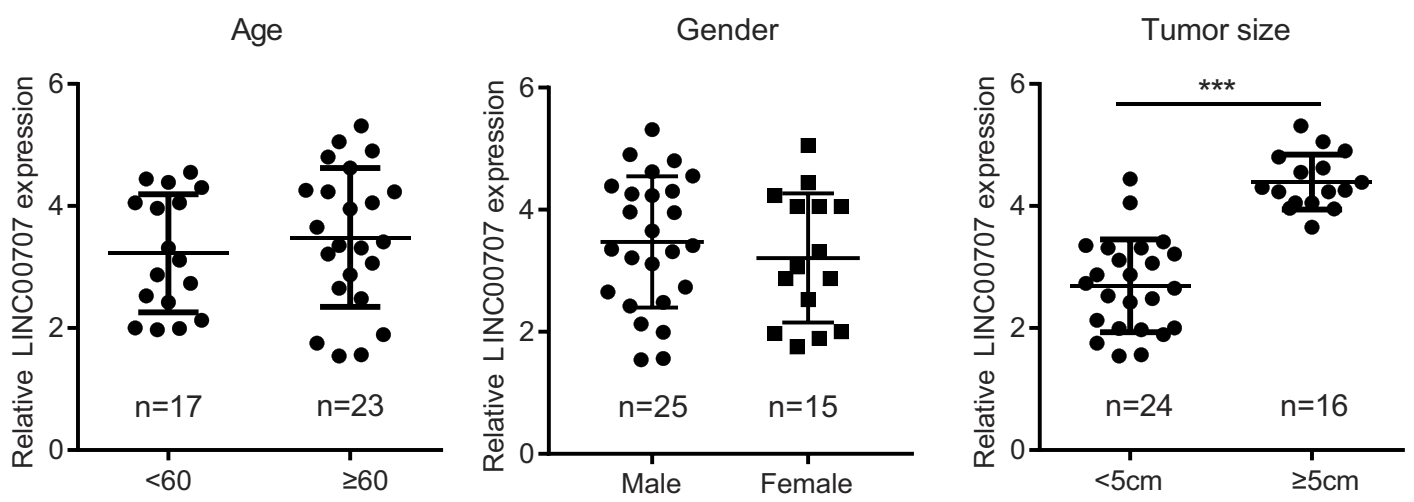

Lymphatic metastasis

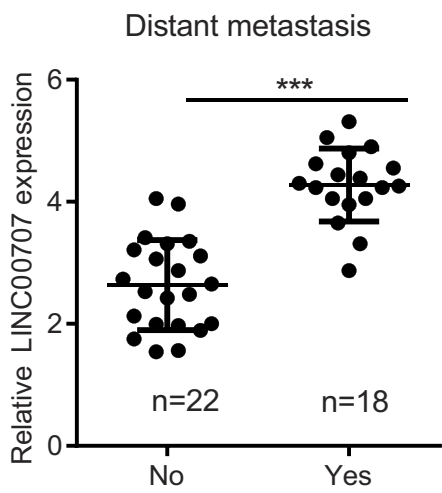

Figure 2 Correlation between LINC00707 expression and clinicopathological characteristics of patients with colorectal cancer. LINC00707 expression was significantly correlated with tumor size, lymphatic metastasis, and distant metastasis, but not significantly correlated with age and gender $(* * * P<0.001)$.

analysis showed that the protein expression of $\mathrm{NOTCH} 3$ and TM4SF1 was downregulated in LoVo and HCT116 cells transfected with si-LINC00707 as compared with the cells transfected with si-NC (Figure 4E).

\section{Discussion}

Studies have demonstrated the important role of lncRNAs in the development and progression of various human cancers, including CRC. Therefore, better understanding of the function and underlying molecular mechanism of lncRNAs may help in the discovery of effective therapeutic strategies for the treatment of patients with CRC. In this study, we found that LINC00707 expression level was significantly upregulated in CRC tissues as compared with the adjacent non-CRC tissues. And LINC00707 expression was significantly correlated with tumor size, lymphatic metastasis, and distant metastasis, but not significantly correlated with age and gender. Knockdown of LINC00707 expression resulted in a significant inhibition of LoVo and HCT116 cell proliferation and metastasis. LINC00707 acted as a molecular sponge by competing for miR-206 and indirectly modulating the expression of its target proteins NOTCH3 and TM4SF1.

Recent studies have indicated the abnormal expression of IncRNAs in CRC. For instance, Lv et al reported that the expression of lncRNA ZEB1-AS1 was markedly upregulated in CRC, and ZEB1-AS1 knockdown inhibited CRC cell proliferation. ${ }^{18}$ In the present study, we found that LINC00707 expression level was significantly upregulated in CRC tissues as compared with adjacent non-CRC tissues and significantly correlated with tumor size, lymphatic metastasis, and distant metastasis. These results indicate that LINC00707 expression may be involved in the development of CRC. Our present study showed that LINC00707 knockdown significantly inhibited LoVo and HCT116 cell growth, migration, and invasion, suggesting that LINC00707 may serve as a potential target for CRC treatment. Our results were consistent with the function of LINC00707 in other cancers. The expression level of LINC00707 was shown to be significantly upregulated in lung adenocarcinoma tissues as compared with the paired normal tissues, and LINC00707 expression correlated with TNM stage, tumor size, lymphatic metastasis. ${ }^{11}$ LINC00707 knockdown resulted in the 
A

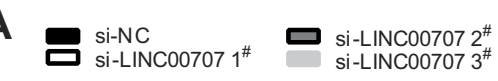
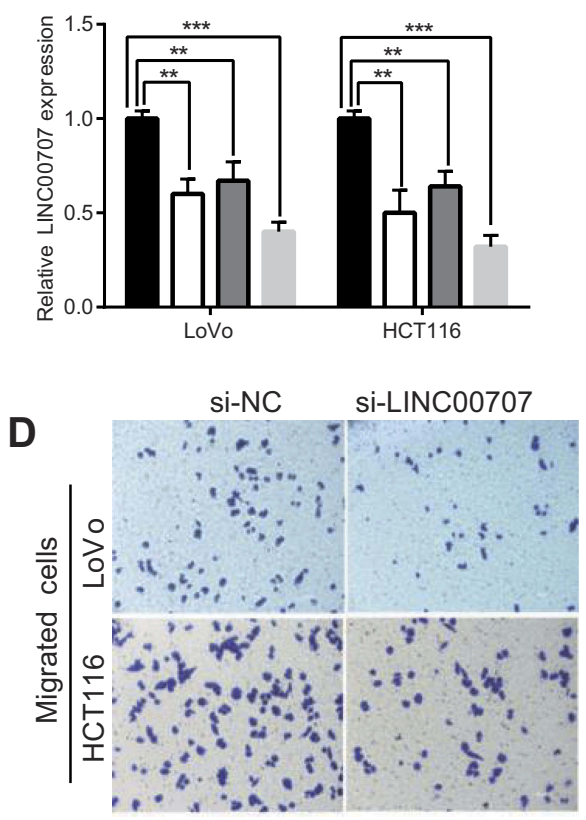

G

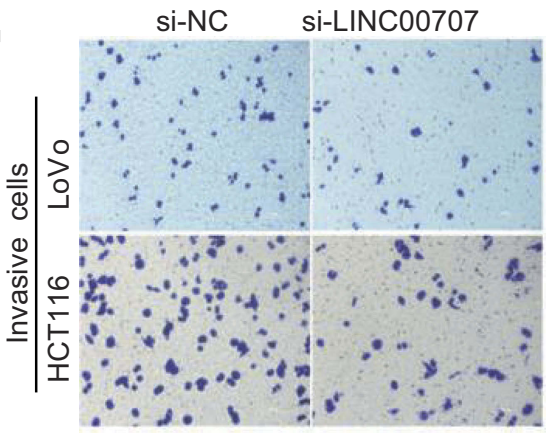

B

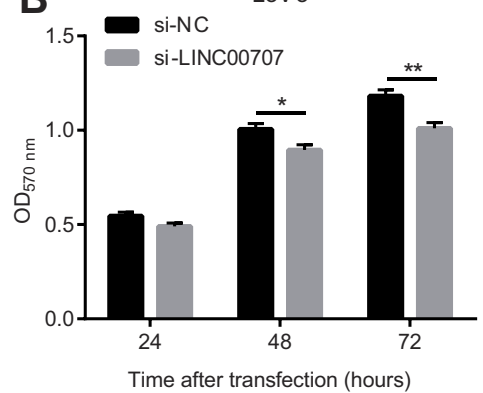

E

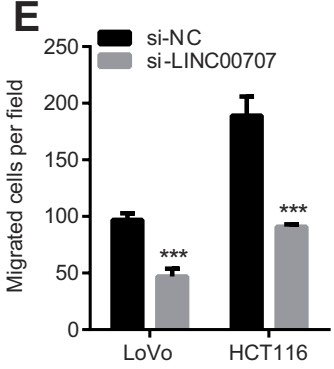

H

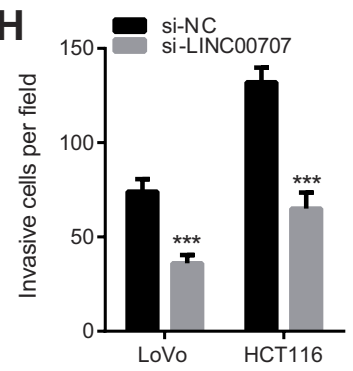

C

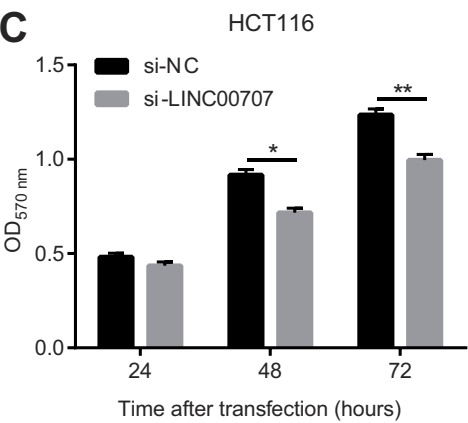

$\mathbf{F}$

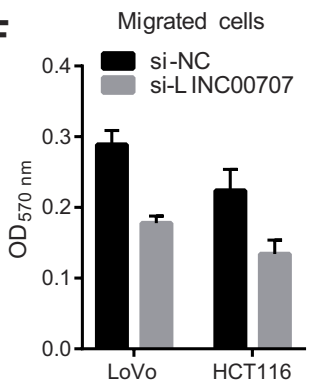

I

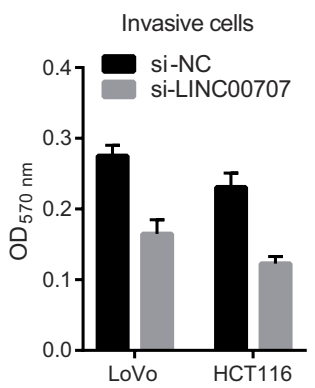

Figure 3 Knockdown of LINC00707 expression inhibits the proliferation, migration, and invasion of colorectal cancer cells. (A) Expression level of LINC00707 in LoVo and HCTII 6 cells transfected with si-LINC00707 I", si-LINC00707 $2^{\#}$, and si-LINC00707 $3^{\#}$, or si-negative control (NC), as analyzed with quantitative reverse transcription PCR. (B and C) Knockdown of LINC00707 expression inhibits LoVo (B) and HCTII6 (C) cell proliferation, as evident in the MTT assay. (D) The representative graphs of migrated cells stained by crystal violet staining in transwell assay. (E) The statistical results of average migrated cells stained by crystal violet in transwell assay. (F) The statistical results of $O_{570 \mathrm{~nm}}$ value of migrated cells determined by MTT assay. (G) The representative graphs of invasive cells stained by crystal violet staining in transwell assay. $(\mathbf{H})$ The statistical results of average invasive cells stained by crystal violet in transwell assay. (I) The statistical results of $\mathrm{OD}_{570 \mathrm{~nm}}$ value of invasive cells determined by MTT assay. $* P<0.05, * * P<0.01$, $* * * P<0.001$.

suppression of lung adenocarcinoma cell proliferation and migration through the regulation of cell division control protein 42 (Cdc42) expression. ${ }^{11}$ In HCC, LINC00707 expression was upregulated, and LINC00707 knockdown inhibited the proliferation of cells through the activation of the extracellular signal-regulated kinase/c-Jun N-terminal kinase/protein kinase B signaling pathway. ${ }^{12}$

Recent studies have revealed that lncRNAs act as ceRNAs and interact with miRNAs. LncRNA H19 promotes cell proliferation by sponging miR-200a, thereby increasing $\beta$-catenin expression in CRC. ${ }^{19}$ LncRNA FTX markedly promoted CRC cell proliferation, migration, and invasion by sponging miR-215. ${ }^{20}$ Several studies have reported that miR-206 acts as a tumor suppressor, and its expression was downregulated in various human malignancies. ${ }^{21,22}$ In CRC, miRNA-206 levels were significantly downregulated in CRC tissues, ${ }^{23,24}$ and miR-206 overexpression inhibited the proliferation, migration, and invasion of CRC cells. ${ }^{24}$ The expression profile and function of miR-206 in CRC is opposite to that of LINC00707. So we predicted that LINC00707 may function as ceRNA of miR-206. Here, our study revealed that miR-206 is a target of LINC00707. Changes in LINC00707 expression led to corresponding changes in the expression levels of miR-206, suggestive of the negative 
A

hsa-miR-206

3'...GUGAAGGAAUGUAAGGUAU...

WT LINC00707

| I| | | | ||

MUT LINC00707

5'...UAAUCAGAAACAUUCCAAC...

5'...UAAUCAGAAAGAUAGGAAC...

B
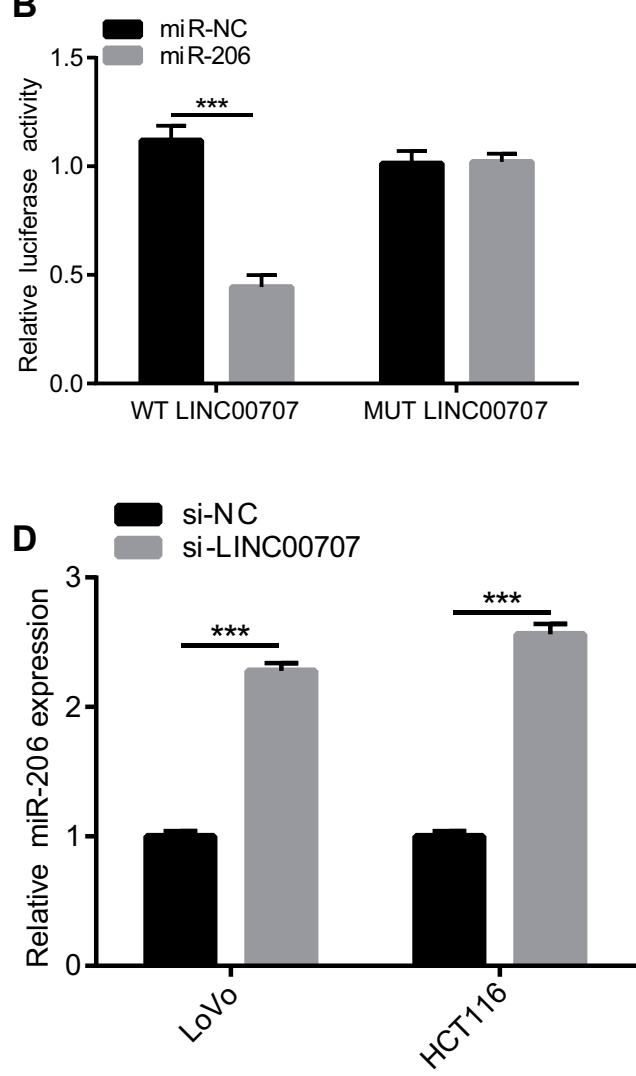

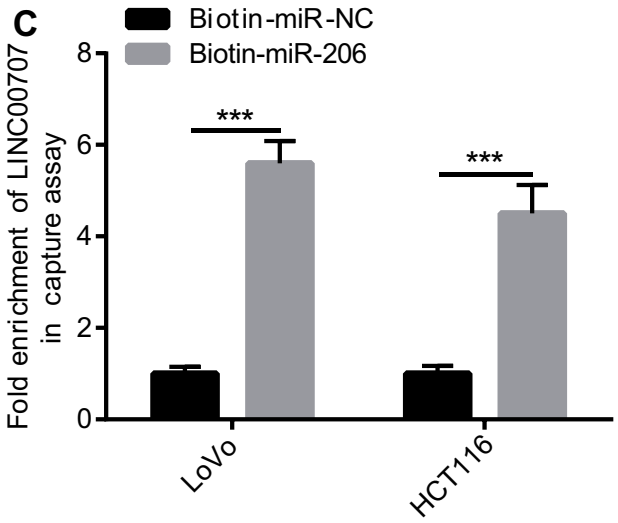

E
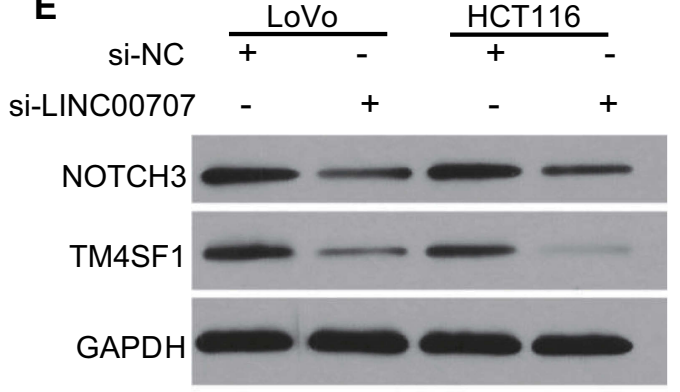

Figure 4 LINC00707 acts as a molecular sponge for miR-206 and indirectly modulates the expression of its targets NOTCH3 and TM4SFI. (A) The predicted binding sites of miR-206 on the sequence of LINC00707. The binding sites were highlighted by capital letter. (B) Luciferase reporter assay was performed to detect luciferase activity after cotransfection of cells with WT-LINC00707 or MUT-LINC00707 and miR-206 mimic. (C) Biotin-coupled miRNA capture assay was performed to measure the expression of LINC00707 in LoVo and HCTII6 cells transfected with biotin-miR-206 or biotin-miR-NC. (D) The expression of miR-206 in LoVo and HCTII6 cells transfected with si-LINC00707 or si-NC was measured with quantitative reverse transcription PCR. (E) The protein expression of NOTCH3 and TM4SFI in LoVo and HCTII 6 cells transfected with si-LINC00707 or si-NC was measured with Western blotting. $* * * P<0.001$.

Abbreviations: MUT, mutant; NC, negative control; WT, wild-type.

regulation of miR-206 expression by LINC00707. Therefore, the effects of LINC00707 on CRC cell proliferation, migration, and invasion may be explained, at least in part, by its role as a molecular sponge of miR-206.

miR-206 inhibits cell proliferation and migration through the downregulation of NOTCH3 expression. ${ }^{15}$ The expression of NOTCH3, identified as the third mammalian notch receptor, was significantly upregulated in $\mathrm{CRC}$, and NOTCH3 silencing suppressed the proliferation and tumorigenic properties of CRC cells. ${ }^{25}$ Park et al reported that miR206 suppresses PGE2-induced cell proliferation, migration, and invasion by targeting TM4SF $1 .{ }^{16} \mathrm{TM} 4 \mathrm{SF} 1$ is a member of the tetraspanin L6 domain family and its expression was shown to be upregulated in primary CRC. In addition,
TM4SF1 regulates the metastatic potential of CRC cells through the activation of epithelial-mesenchymal transition regulator. ${ }^{26}$ These reports have indicated that NOTCH3 and TM4SF1 are targets of miR-206 and act as important regulators in the development and metastases of CRC. In our study, we found that LINC00707 knockdown significantly decreased the protein expression of NOTCH3 and TM4SF 1 in both LoVo and HCT116 cells. These results further indicated that LINC00707 promotes CRC cell proliferation and metastasis by sponging miR-206.

The present study has some limitations. Follow-up study was not carried out in present study. The potential prognostic role of LINC00707 should be evaluated in the further study. Although we identified miR-206 as a target of LINC00707, 
LINC00707 may have other potential targets. Further research is warranted to study how LINC00707 differentiates and regulates these microRNAs. Moreover, miR-206 has many target genes, including the target genes we detected. Furthermore, we did not verify the expression profile of LINC00707 in CRC cell lines and the effect of LINC00707 knockdown on tumor inhibition in animals. Despite these drawbacks, our study highlights the potential role of LINC00707 in CRC development and metastasis.

In conclusion, we demonstrate the high expression of LINC00707 in CRC tissues. Knockdown of LINC00707 expression significantly inhibits CRC cell growth and metastasis by sponging miR-206. These findings suggest that LINC00707 may serve as a potential target for CRC treatment.

\section{Acknowledgments}

The work was supported by the National Natural Science Foundation of China (grant numbers 31800658, 81772524, and 81802470) and the Science and Technology Innovation Foundation of Xinxiang (CXTD17005).

\section{Disclosure}

The authors declare that they have no competing interests.

\section{References}

1. Torre LA, Bray F, Siegel RL, Ferlay J, Lortet-Tieulent J, Jemal A. Global cancer statistics, 2012. CA Cancer J Clin. 2015;65(2):87-108. doi:10.3322/caac. 21262

2. Chen W, Zheng R, Baade PD, et al. Cancer statistics in China, 2015. CA Cancer J Clin. 2016;66(2):115-132. doi:10.3322/caac.21338

3. Chen W, Zheng R, Zhang S, Zhao P, Zeng H, Zou X. Report of cancer incidence and mortality in China, 2010. Ann Transl Med. 2014;2(7):61.

4. Fatica A, Bozzoni I. Long non-coding RNAs: new players in cell differentiation and development. Nat Rev Genet. 2014;15(1):7-21. doi: $10.1038 / \operatorname{nrg} 3606$

5. Yang S, Sun Z, Zhou Q, et al. MicroRNAs, long noncoding RNAs, and circular RNAs: potential tumor biomarkers and targets for colorectal cancer. Cancer Manag Res. 2018;10:2249-2257. doi:10.2147/CMAR. S166308

6. Jarroux J, Morillon A, Pinskaya M. History, discovery, and classification of lncRNAs. Adv Exp Med Biol. 2017;1008:1-46. doi:10.1007/ 978-981-10-5203-3_1

7. Saha P, Verma S, Pathak RU, Mishra RK. Long noncoding RNAs in mammalian development and diseases. Adv Exp Med Biol. 2017;1008:155-198. doi:10.1007/978-981-10-5203-3_6

8. Shan Y, Ma J, Pan Y, Hu J, Liu B, Jia L. LncRNA SNHG7 sponges miR-216b to promote proliferation and liver metastasis of colorectal cancer through upregulating GALNT1. Cell Death Dis. 2018;9(7):722. doi: 10.1038/s41419-018-0759-7

9. Ji Q, Liu X, Fu X, et al. Resveratrol inhibits invasion and metastasis of colorectal cancer cells via MALAT1 mediated $\mathrm{Wnt} / \beta$-catenin signal pathway. PLoS One. 2013;8(11):e78700. doi:10.1371/journal. pone. 0078700
10. Bian Z, Jin L, Zhang J, et al. LncRNA-UCA1 enhances cell proliferation and 5-fluorouracil resistance in colorectal cancer by inhibiting miR-204-5p. Sci Rep. 2016;6:23892. doi:10.1038/ srep23892

11. Ma T, Ma H, Zou Z, et al. The long intergenic noncoding RNA 00707 promotes lung adenocarcinoma cell proliferation and migration by regulating Cdc42. Cell Physiol Biochem. 2018;45(4):1566-1580. doi: $10.1159 / 000487693$

12. Wang J, Luo Z, Yao T, Li W, Pu J. LINC00707 promotes hepatocellular carcinoma progression through activating ERK/JNK/AKT pathway signaling pathway. J Cell Physiol. 2019:234(5):6908-6916. doi: $10.1002 /$ jcp. 27449

13. Cesana M, Cacchiarelli D, Legnini I, et al. A long noncoding RNA controls muscle differentiation by functioning as a competing endogenous RNA. Cell. 2011;147(2):358-369. doi:10.1016/j. cell.2011.09.028

14. Oliveto S, Mancino M, Manfrini N, Biffo S. Role of microRNAs in translation regulation and cancer. World J Biol Chem. 2017;8 (1):45-56. doi:10.4331/wjbc.v8.i1.45

15. Wang X-W, Xi X-Q, Wu J, Wan Y-Y, Hui H-X, Cao X-F. MicroRNA206 attenuates tumor proliferation and migration involving the downregulation of NOTCH3 in colorectal cancer. Oncol Rep. 2015;33 (3):1402-1410. doi:10.3892/or.2015.3731

16. Park YR, Seo SY, Kim SL, et al. MiRNA-206 suppresses PGE2-induced colorectal cancer cell proliferation, migration, and invasion by targetting TM4SF1. Biosci Rep. 2018;38(5): BSR20180664. doi:10.1042/BSR20180664

17. Yang C, Yuan W, Yang X, et al. Circular RNA circ-ITCH inhibits bladder cancer progression by sponging miR-17/miR-224 and regulating p21, PTEN expression. Mol Cancer. 2018;17(1):19. doi:10.1186/s12943-018-0771-7

18. Lv S-Y, Shan T-D, Pan X-T, et al. The lncRNA ZEB1-AS1 sponges miR-181a-5p to promote colorectal cancer cell proliferation by regulating Wnt/ $\beta$-catenin signaling. Cell Cycle. 2018;17(10):1245-1254. doi:10.1080/15384101.2018.1471317

19. Yang W, Ning N, Jin X. The lncRNA H19 promotes cell proliferation by competitively binding to miR-200a and derepressing beta-catenin expression in colorectal cancer. Biomed Res Int. 2017;2017:2767484.

20. Yang Y, Zhang J, Chen X, et al. LncRNA FTX sponges miR-215 and inhibits phosphorylation of vimentin for promoting colorectal cancer progression. Gene Ther. 2018;25(5):321-330. doi:10.1038/s41434-0180026-7

21. Kondo N, Toyama T, Sugiura H, Fujii Y, Yamashita H. miR-206 expression is down-regulated in estrogen receptor alpha-positive human breast cancer. Cancer Res. 2008;68(13):5004-5008. doi:10.1158/0008-5472.CAN-08-0180

22. Zhang L, Liu X, Jin H, et al. miR-206 inhibits gastric cancer proliferation in part by repressing cyclinD2. Cancer Lett. 2013;332 (1):94-101. doi:10.1016/j.canlet.2013.01.023

23. Sun P, Sun D, Wang X, Liu T, Ma Z, Duan L. miR-206 is an independent prognostic factor and inhibits tumor invasion and migration in colorectal cancer. Cancer Biomark. 2015;15(4):391-396. doi:10.3233/CBM-150489

24. Ren XL, He GY, Li XM, et al. MicroRNA-206 functions as a tumor suppressor in colorectal cancer by targeting FMNL2. $J$ Cancer Res Clin Oncol. 2016;142(3):581-592. doi:10.1007/ s00432-015-2053-8

25. Serafin V, Persano L, Moserle L, et al. Notch3 signalling promotes tumour growth in colorectal cancer. $J$ Pathol. 2011;224(4):448-460. doi:10.1002/path.2895

26. Park YR, Kim SL, Lee MR, et al. MicroRNA-30a-5p (miR-30a) regulates cell motility and EMT by directly targeting oncogenic TM4SF1 in colorectal cancer. J Cancer Res Clin Oncol. 2017;143 (10):1915-1927. doi:10.1007/s00432-017-2440-4 


\section{Supplementary material}

Table SI The clinical information of the 40 patients analyzed in the study

\begin{tabular}{|c|c|c|c|c|c|}
\hline Patient no. & Age (years) & Gender & Tumor size & Lymphatic metastasis & Distant metastasis \\
\hline I & 45 & Female & $<5 \mathrm{~cm}$ & No & No \\
\hline 2 & 65 & Male & $<5 \mathrm{~cm}$ & No & No \\
\hline 3 & 38 & Male & $<5 \mathrm{~cm}$ & No & No \\
\hline 4 & 59 & Female & $<5 \mathrm{~cm}$ & No & No \\
\hline 5 & 37 & Female & $<5 \mathrm{~cm}$ & No & No \\
\hline 6 & 46 & Male & $<5 \mathrm{~cm}$ & No & No \\
\hline 7 & 44 & Female & $\geq 5 \mathrm{~cm}$ & No & No \\
\hline 8 & 40 & Male & $<5 \mathrm{~cm}$ & No & No \\
\hline 9 & 56 & Male & $\geq 5 \mathrm{~cm}$ & Yes & Yes \\
\hline 10 & 31 & Female & $\geq 5 \mathrm{~cm}$ & No & Yes \\
\hline II & 69 & Male & $\geq 5 \mathrm{~cm}$ & Yes & Yes \\
\hline 12 & 59 & Male & $<5 \mathrm{~cm}$ & No & No \\
\hline 13 & 43 & Male & $\geq 5 \mathrm{~cm}$ & Yes & No \\
\hline 14 & 40 & Female & $<5 \mathrm{~cm}$ & No & Yes \\
\hline 15 & 76 & Male & $\geq 5 \mathrm{~cm}$ & Yes & Yes \\
\hline 16 & 32 & Male & $<5 \mathrm{~cm}$ & No & No \\
\hline 17 & 45 & Male & $<5 \mathrm{~cm}$ & Yes & No \\
\hline 18 & 65 & Female & $<5 \mathrm{~cm}$ & No & No \\
\hline 19 & 61 & Male & $<5 \mathrm{~cm}$ & Yes & No \\
\hline 20 & 68 & Male & $\geq 5 \mathrm{~cm}$ & Yes & Yes \\
\hline 21 & 60 & Male & $\geq 5 \mathrm{~cm}$ & Yes & Yes \\
\hline 22 & 64 & Female & $<5 \mathrm{~cm}$ & No & Yes \\
\hline 23 & 68 & Male & $<5 \mathrm{~cm}$ & Yes & No \\
\hline 24 & 63 & Male & $<5 \mathrm{~cm}$ & Yes & Yes \\
\hline 25 & 38 & Female & $<5 \mathrm{~cm}$ & No & No \\
\hline 26 & 65 & Male & $<5 \mathrm{~cm}$ & No & No \\
\hline 27 & 61 & Male & $\geq 5 \mathrm{~cm}$ & Yes & Yes \\
\hline 28 & 36 & Male & $\geq 5 \mathrm{~cm}$ & Yes & No \\
\hline 29 & 72 & Female & $<5 \mathrm{~cm}$ & No & No \\
\hline 30 & 70 & Male & $\geq 5 \mathrm{~cm}$ & Yes & Yes \\
\hline 31 & 62 & Female & $\geq 5 \mathrm{~cm}$ & Yes & Yes \\
\hline 32 & 69 & Female & $<5 \mathrm{~cm}$ & No & Yes \\
\hline 33 & 67 & Male & $<5 \mathrm{~cm}$ & Yes & No \\
\hline 34 & 60 & Female & $<5 \mathrm{~cm}$ & No & No \\
\hline 35 & 54 & Female & $<5 \mathrm{~cm}$ & No & No \\
\hline 36 & 64 & Male & $\geq 5 \mathrm{~cm}$ & Yes & Yes \\
\hline 37 & 63 & Male & $\geq 5 \mathrm{~cm}$ & Yes & Yes \\
\hline 38 & 68 & Female & $\geq 5 \mathrm{~cm}$ & Yes & Yes \\
\hline 39 & 60 & Male & $\geq 5 \mathrm{~cm}$ & Yes & Yes \\
\hline 40 & 70 & Male & $<5 \mathrm{~cm}$ & No & No \\
\hline
\end{tabular}




\section{Publish your work in this journal}

OncoTargets and Therapy is an international, peer-reviewed, open access journal focusing on the pathological basis of all cancers, potential targets for therapy and treatment protocols employed to improve the management of cancer patients. The journal also focuses on the impact of management programs and new therapeutic agents and protocols on patient perspectives such as quality of life, adherence and satisfaction. The manuscript management system is completely online and includes a very quick and fair peer-review system, which is all easy to use. Visit http://www.dovepress.com/ testimonials.php to read real quotes from published authors. 